\title{
Validity of the Surveillance Quality Indicators - Timeliness and Completeness - in Surveillance Systems with Variable Data Quality
}

\author{
Vivek Singh*1, Jagan Mohan², U Prasada Rao², Lalit Dandona ${ }^{3,4}$ and David Heymann ${ }^{5}$ \\ ${ }^{1}$ Public Health Foundation of India, Indian Institute of Public Health - Hyderabad, Hyderabad, India; ${ }^{2}$ Directorate of Health, \\ Department of Health, Medical \& Family Welfare, Government of Andhra Pradesh, Hyderabad, India; ${ }^{3}$ Public Health Foundation of \\ India, New Delhi, India; ${ }^{4}$ Institute for Health Metrics and Evaluation, University of Washington, Seattle, WA, USA; ${ }^{5}$ London School of \\ Hygiene and Tropical Medicine, London, United Kingdom
}

\section{Objective}

To assess the validity of the Surveillance Quality Indicators (SQIs), Timeliness and Completeness of the weekly surveillance reports as indicators for overall quality of surveillance system and core and supplementary surveillance functions

\section{Introduction}

India is one of the global 'hot-spots' for emergence and re-emergence of pathogens and propagation of those that are drug resistant. Disease surveillance gained momentum in India only after the outbreaks of cholera in Delhi in 1988 and plague in Surat in 1994, which not only had significant mortality, morbidity and economic consequences. The current key indicator based surveillance system in the country, the Integrated Disease Surveillance Project (IDSP) has evolved from systems that were initiated and scaled up as a response to these outbreaks. IDSP is constrained by challenges of human and material resources and the quality of data generated at the frontline is questionable making it difficult to detect, diagnose, and control outbreaks until they had become quite large. Timeliness and Completeness of weekly reports are the two key SQIs even suggested by the World Health Organization (WHO) to monitor the quality of the surveillance system in the districts and states. The goal of the current study was to assess the validity of these SQIs in predicting the overall surveillance quality in a system where data quality was questionable.

\section{Methods}

A cross-sectional survey was undertaken involving all 23 districts in the state of Andhra Pradesh in India. A composite score derived from Timeliness and Completeness of the weekly surveillance reports was used to select 6 districts for visiting District Surveillance Units and 35 health facilities for conducting 110 Key Informant Interviews (KIIs), direct observations and secondary data analysis of surveillance records. The districts with composite score $=$ or $>60$ were categorized as good and those with score $<60$ were categorized as poor. The key surveillance system attributes and core and supplementary surveillance functions were compared in the two groups of districts. The framework for the assessment was adapted from the frameworks proposed by the WHO and by Scott McNabb et al. for evaluation of surveillance systems.

\section{Results}

Based on the composite score, 8 districts were in the Good SQI group and 15 districts were in the Poor SQI group. The difference in the mean average Completeness scores (20.21) and the Composite scores (16.06) between the two groups were statistically significant $\mathrm{p}<0.01$ however there was no statistically significant difference between the mean Timeliness scores (3.56) of the two groups. There was no statistically significant difference in the core and support surveillance functions in the Good and Poor SQI districts groups.

\section{Conclusions}

Validity of Timeliness and Completeness of weekly surveillance reports, as SQIs is questionable due to poor data quality - evident from the finding that there was no statistically significant difference in the core and support surveillance functions in the Good and Poor SQI groups. Maturing surveillance systems that have variable data quality should have more comprehensive indicators - including all system level and core and support surveillance functions - for monitoring surveillance quality.

\section{Keywords}

Surveillance Quality Indicators; Maturing Surveillance Systems; Poor Data Quality; Comprehensive Surveillance Quality Indicators

\section{Acknowledgments}

Authors acknowledge the participation of government health functionaries from the department of health, medical and family welfare, Government of Andhra Pradesh. This work was part of a fellowship grant through the Wellcome Trust Capacity Strengthening Strategic Award to the PHFI and consortium of UK Universities.

\section{References}

1. India - Integrated Disease Surveillance Project - Project Information Document. The World Bank, 2001 Contract No.: PID10512.

2. Suresh K. Integrated Diseases Surveillance Project (IDSP) through a consultant's lens. Indian journal of public health. 2008 JulSep;52(3):136-43. PubMed PMID: 19189835.

3. John TJ, Dandona L, Sharma VP, Kakkar M. Continuing challenge of infectious diseases in India. Lancet. 2011 Jan 15;377(9761):252-69. PubMed PMID: 21227500.

4. World Health Organization: Communicable disease surveillance and response system - guide to monitoring and evaluating World Health Organization, 2006.

5. McNabb SJ, Chungong S, Ryan M, Wuhib T, Nsubuga P, Alemu W, et al. Conceptual framework of public health surveillance and action and its application in health sector reform BMC public health. 2002;2:2. PubMed PMID: 11846889. Pubmed Central PMCID: 65598.

6. Heymann DL, Rodier GR; WHO Operational Support Team to the Global Outbreak Alert and Response Network. Hot spots in a wired world: WHO surveillance of emerging and re-emerging infectious diseases. The Lancet Infectious Diseases, 2001, 1:345-353. doi:10.1016/ S1473-3099(01)00148-7 pmid:11871807

*Vivek Singh

E-mail: vivek.singh@iiphh.org 